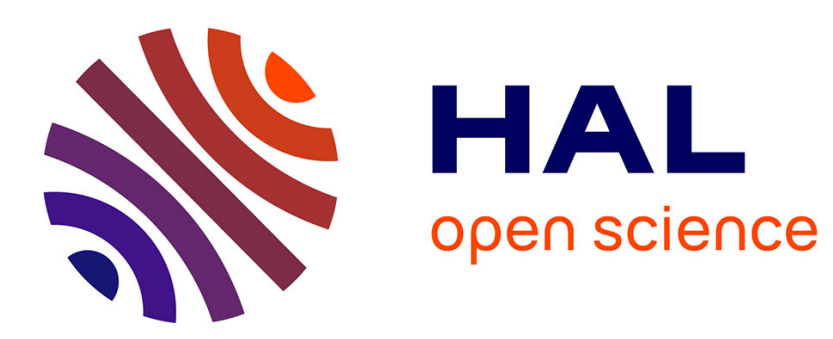

\title{
The Normal Graph Conjecture for Two Classes of Sparse Graphs
}

Anne Berry, Annegret K Wagler

\section{To cite this version:}

Anne Berry, Annegret K Wagler. The Normal Graph Conjecture for Two Classes of Sparse Graphs. Graphs and Combinatorics, 2018, 34 (1), pp.139-157. hal-02045757

\section{HAL Id: hal-02045757 https://hal.science/hal-02045757}

Submitted on 22 Feb 2019

HAL is a multi-disciplinary open access archive for the deposit and dissemination of scientific research documents, whether they are published or not. The documents may come from teaching and research institutions in France or abroad, or from public or private research centers.
L'archive ouverte pluridisciplinaire HAL, est destinée au dépôt et à la diffusion de documents scientifiques de niveau recherche, publiés ou non, émanant des établissements d'enseignement et de recherche français ou étrangers, des laboratoires publics ou privés. 


\title{
The Normal Graph Conjecture for two classes of sparse graphs
}

\author{
Anne Berry • Annegret K. Wagler
}

Received: date / Accepted: date

\begin{abstract}
Normal graphs are defined in terms of cross-intersecting set families: a graph is normal if it admits a clique cover $\mathcal{Q}$ and a stable set cover $\mathcal{S}$ s.t. every clique in $\mathcal{Q}$ intersects every stable set in $\mathcal{S}$. Normal graphs can be considered as closure of perfect graphs by means of co-normal products and graph entropy. Perfect graphs have been characterized as those graphs without odd holes and odd antiholes as induced subgraphs (Strong Perfect Graph Theorem, Chudnovsky et al. 2002). Körner and de Simone observed that $C_{5}$, $C_{7}$, and $\bar{C}_{7}$ are minimal not normal and conjectured, in analogy to the Strong Perfect Graph Theorem, that every $\left(C_{5}, C_{7}, \bar{C}_{7}\right)$-free graph is normal (Normal Graph Conjecture, Körner and de Simone 1999). Recently, this conjecture has been disproved by Harutyunyan, Pastor and Thomassé. However, in this paper we verify it for two classes of sparse graphs, 1-trees and cacti. In addition, we provide both linear time recognition algorithms and characterizations for the normal graphs within these two classes.
\end{abstract}

Keywords Normal Graph Conjecture · 1-trees · cacti

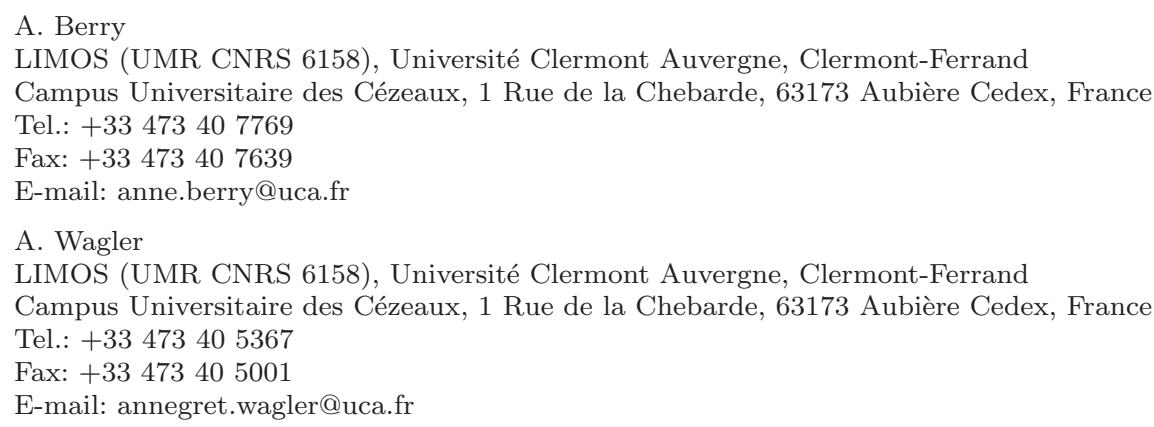




\section{Introduction}

Normal graphs come up in a natural way in an information theoretic context by studying co-normal products [7] or graph entropies [4]. A graph $G$ is normal if $G$ admits cross-intersecting clique and stable set covers, called a valid pair $(\mathcal{Q}, \mathcal{S})$ : a clique cover $\mathcal{Q}$ and a stable set cover $\mathcal{S}$ s.t. every clique in $\mathcal{Q}$ intersects every stable set in $\mathcal{S}$. (A set is a clique (resp. stable set) if its nodes are mutually adjacent (resp. non-adjacent).) Figure 1 presents three normal graphs and their valid pairs.
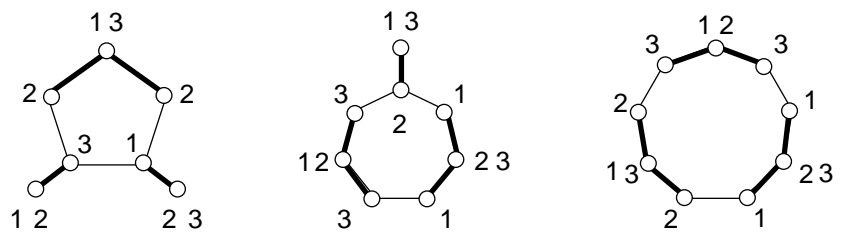

Fig. 1 Three normal graphs and their valid pairs: bold edges indicate the clique covers, labels the cross-intersecting stable set covers

The class of normal graphs includes the well-studied perfect graphs, and the interest in normal graphs is caused by the fact that they form, in different ways, a weaker variant of perfect graphs.

Berge introduced the latter class in 1960, motivated from Shannon's information-theoretic problem of finding the zero-error capacity of a discrete memoryless channel [14]. Shannon's problem has a graph-theoretic formulation, regarding the asymptotic growth of the maximum cliques in the co-normal product $G^{n}$ of $G=(V, E)$, where $G^{2}$ has $V \times V$ as node set and $\left\{\left(a_{1}, b_{1}\right),\left(a_{2}, b_{2}\right)\right.$ : $\left(a_{1}, a_{2}\right) \in E$ or $\left.\left(b_{1}, b_{2}\right) \in E\right\}$ as edge set.

Berge [1] introduced perfect graphs as those graphs $G$, where the clique number $\omega\left(G^{\prime}\right)$ equals the chromatic number $\chi\left(G^{\prime}\right)$ for each induced subgraph $G^{\prime} \subseteq G(\omega(G)$ denotes the size of a maximum clique in $G, \chi(G)$ the least number of stable sets covering all nodes of $G$ ).

Berge observed that chordless odd cycles $C_{2 k+1}$ with $k \geq 2$, the odd holes, and their complements, the odd antiholes $\bar{C}_{2 k+1}$ with $k \geq 2$, are graphs $G$ with $\omega(G)<\chi(G)$, see Figure 2. (The complement $\bar{G}$ has the same node set as $G$ but two nodes are adjacent in $\bar{G}$ iff they are non-adjacent in $G$.) This motivated Berge's Strong Perfect Graph Conjecture: a graph $G$ is perfect if and only if $G$ is odd hole- and odd antihole-free. In a sequence of remarkable results, Chudnovsky et al. [3] finally turned the above conjecture into the Strong Perfect Graph Theorem.

Normal graphs are a closure of perfect graphs in terms of graph entropy $[4,8,15]$ and taking co-normal products: Körner and Longo [7] showed that all co-normal products of a graph $G$ are perfect only if $G$ is the union of disjoint cliques. However, all co-normal products of normal (and, therefore, of perfect) graphs are normal by [6]. 

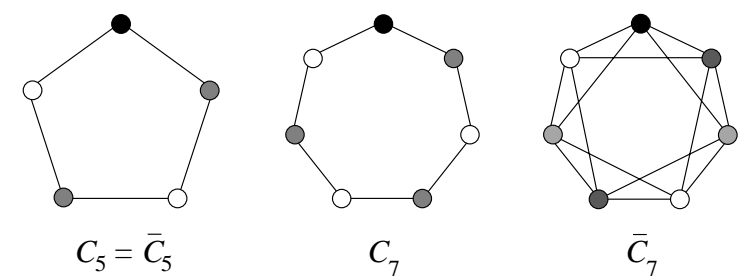

Fig. 2 Small odd holes and odd antiholes

This motivated Körner and de Simone [9] to ask for a similarity of the two classes in terms of forbidden subgraphs. Körner [6] showed that an odd hole $C_{2 k+1}$ is normal if and only if $k \geq 4$. By the invariance of normality under taking complements, an odd antihole $\bar{C}_{2 k+1}$ is also normal if and only if $k \geq 4 . C_{5}, C_{7}$ and $\bar{C}_{7}$ are minimally not normal since all of their proper induced subgraphs are perfect and, hence, normal. Körner and de Simone conjectured that there are no other minimally not normal graphs:

Conjecture 1 (Normal Graph Conjecture [9]) All graphs without a $C_{5}, C_{7}$, and $\bar{C}_{7}$ as induced subgraph are normal.

Note that the non-existence of $C_{5}, C_{7}$, and $\bar{C}_{7}$ in a graph is not necessary to be normal (see the first two graphs in Figure 1), and a characterization of normal graphs by forbidden subgraphs is not possible (see [17]). So far, the Normal Graph Conjecture has been verified for webs [16] and line graphs [12].

In order to treat the Strong Perfect Graph Conjecture from a probabilistic point of view, Prömel and Steger [11] asked for the relation of perfect and odd hole, odd antihole-free graphs with the same number of nodes. For that, they proved that almost all $C_{5}$-free graphs are perfect. Since every $\left(C_{5}, C_{7}, \bar{C}_{7}\right)$ free graph is $C_{5}$-free, also almost all $\left(C_{5}, C_{7}, \bar{C}_{7}\right)$-free graphs are perfect and, therefore, normal.

However, the Normal Graph Conjecture has been recently disproved by Harutyunyan, Pastor and Thomassé [5]. Their proof is based on probabilistic arguments, showing the existence of a not normal graph with girth at least 8 , i.e., without holes $C_{k}$ of length $k \leq 7$ (which also excludes $\bar{C}_{7}$ since it contains $C_{3}$ and $C_{4}$ ), but without presenting an explicit counterexample.

Both results together imply that there are only few counterexamples and that they should be sparse (due to the large girth $\geq 8$ ). This motivates our study of two classes of sparse graphs w.r.t. normality.

We start with a class of graphs having as many edges as nodes: A 1-tree is a connected graph $G=(V, E)$ with $|V|=|E|$ (obtained from a tree by adding one edge since trees are precisely the connected graphs with $|V|-1=|E|)$. Hence, $G$ contains exactly one cycle $C$ (due to this property, 1-trees are also called unicyclic graphs). In other words, $G$ can be obtained from the cycle $C$ and certain trees by a sequence of node-identifications.

In Section 3, we characterize the normal 1-trees with the help of two results: a connected triangle-free graph is normal if and only if it has a so-called 
nice edge cover [9] and the identification of two normal graphs in one node yields a normal graph again. The latter is a consequence from results on clique identification and normality in [17], see Section 2 . We obtain that the only not normal 1-trees are either equal to a $C_{7}$ or contain a $C_{5}$. This verifies the Normal Graph Conjecture for the class of 1-trees (note: no $\bar{C}_{7}$ can occur in a 1-tree, so it suffices to show that $\left(C_{5}, C_{7}\right)$-free 1 -trees are normal) and leads to a linear time recognition algorithm for normal 1-trees.

We then use these results to treat the larger class of cacti in Section 4. A cactus is a connected graph whose cycles are all edge-disjoint. Thus, a cactus $G=(V, E)$ with $k$ cycles can be considered as a graph obtained from a tree by adding $k$ edges in a certain way (thus cacti admit $|V|-1+k$ edges) or, alternatively, as a graph obtained from $k 1$-trees by a sequence of nodeidentifications.

We can, therefore, apply the characterization of normal 1-trees from Section 3 in order to decide whether or not a cactus is normal: As the class of normal graphs is closed under node identification (see Section 2), a cactus is normal if it can be obtained by identifying normal 1-trees in nodes; this verifies the Normal Graph Conjecture for cacti, as in particular all $\left(C_{5}, C_{7}\right)$-free cacti are normal.

Moereover, there also exist normal cacti containing a $C_{5}$ or a $C_{7}$, so our further goal is to recognize normal cacti. As one can obtain a normal graph by identifying certain not normal graphs in one node, see Section 2 again, we examine in detail when a graph constructed by identifying two 1-trees in a node can be normal. This enables us to design an algorithm that decides for a cactus, given a decomposition into 1-trees, in linear time whether or not it is normal. In addition, we achieve a characterization of normal cacti.

We close with some concluding remarks and discuss consequences and some future lines of research.

Parts of the here presented results appeared without proofs in [2].

\section{Clique identification and normal graphs}

A graph $G$ arises by identification of two disjoint graphs $G_{1}$ and $G_{2}$ in a clique if there are cliques $Q_{1} \subseteq G_{1}$ and $Q_{2} \subseteq G_{2}$ with $\left|Q_{1}\right|=\left|Q_{2}\right|$ and a bijection $\phi: Q_{1} \rightarrow Q_{2}$ identifying every node $v \in Q_{1}$ with $\phi(v) \in Q_{2}$, see Figure 3 .

In this section, we discuss how normal graphs can be obtained by clique identification. The first two graphs in Figure 1 are examples of normal graphs, constructed by identifying a non-normal $C_{5}$ and $C_{7}$ with two edges and one edge, respectively. Figure 3 shows another normal graph obtained by identifying two non-normal graphs in an edge.

However, the non-normal building blocks in these examples are not too far from being normal which shows in particular that it obviously suffices if the nodes in the common clique are covered in one of the two building blocks. This suggests to relax the condition of normality for the building blocks as follows. Let $G$ be a graph such that $G$ has a stable set cover $\mathcal{S}, G-Q^{\prime}$ has 


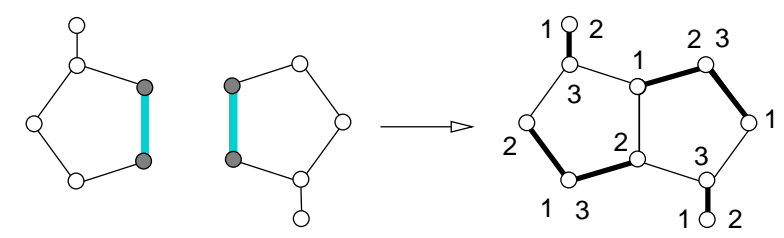

Fig. 3 Identifying two non-normal graphs in an edge: bold edges indicate the clique cover, labels the cross-intersecting stable set cover of the resulting normal graph

a clique cover $\mathcal{Q}_{Q^{\prime}}$, for some clique $Q^{\prime}$, and $\mathcal{S}$ and $\mathcal{Q}_{Q^{\prime}}$ are cross-intersecting. We call such a graph $G$ nearly normal, $\left(\mathcal{Q}_{Q^{\prime}}, \mathcal{S}\right)$ a nearly valid pair of $G$, and $Q^{\prime}$ an unnormal clique of $G$. Figure 4 shows examples of nearly normal graphs together with their nearly valid pairs and unnormal cliques.
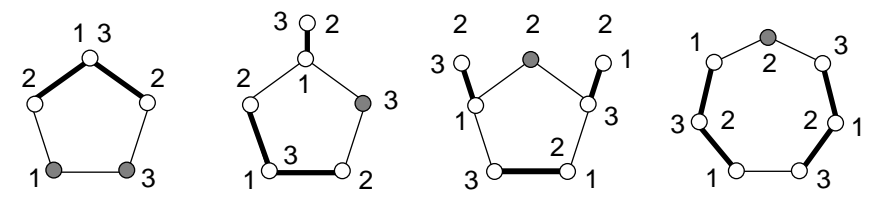

Fig. 4 Nearly normal graphs: the gray-shaded nodes induce the unnormal cliques $Q^{\prime}$, bold edges indicate the clique covers $\mathcal{Q}_{Q^{\prime}}$, labels the cross-intersecting stable set covers

It was shown in [17] that this weaker form of normality suffices for constructing a normal graph by clique identification, provided the involved stable set covers are suitable.

Lemma 1 [17] Construct $G$ by identifying two nearly normal graphs $G_{1}$ and $G_{2}$ in a clique $Q^{*}$ and let $Q_{1}, Q_{2} \subseteq Q^{*}$ be disjoint unnormal cliques. The resulting graph $G$ is normal if there exist nearly valid pairs $\left(\mathcal{Q}_{Q_{i}}\left(G_{i}\right), \mathcal{S}\left(G_{i}\right)\right)$ for $i=1,2$ satisfying at least one of the following conditions.

(1) $\mathcal{S}\left(G_{i}\right)$ contains a stable set $S$ with $S \cap Q^{*}=\emptyset$ for $i=1,2$;

(2) $\mathcal{S}\left(G_{i}\right)$ contains no stable set $S$ with $S \cap Q^{*}=\emptyset$ for $i=1,2$;

(3) $\mathcal{S}\left(G_{1}\right)$ contains a stable set $S$ with $S \cap Q^{*}=\emptyset$ but $\mathcal{S}\left(G_{2}\right)$ does not, and $Q_{1}$

is non-empty (or vice versa).

Lemma 1 provides sufficient conditions to obtain a normal graph via clique identification, but it is not yet clear whether these conditions are also necessary. However, condition (1) of Lemma 1 is certainly satisfied if $Q^{*}$ is a non-maximal clique in $G_{i}$ for $i=1,2$ : In order to cover a common neighbor $v$ of all nodes in $Q^{*}, \mathcal{S}\left(G_{i}\right)$ has to contain a stable set $S$ with $v \in S$ and, thus, $S \cap Q^{*}=\emptyset$.

Corollary 1 The class of normal graphs is closed under identification in nonmaximal cliques. 
In particular, this is the case if $Q^{*}$ consists in a single (but not isolated) node only. Thus, the class of normal graphs is closed under node-identification.

As we have seen that we can construct normal graphs by identifying two unnormal ones in a clique (as in Figure 3), a natural question is whether there exist further ways to construct normal graphs by clique identification. The next lemma from [17] gives an answer showing that the normality or nearnormality of the building blocks is required if the resulting graph is supposed to be normal.

Lemma 2 [17] Let $G$ be a normal graph obtained by identifying two graphs $G_{1}$ and $G_{2}$ in a clique $Q^{*}$.

(1) If $G$ admits a valid pair $(\mathcal{Q}, \mathcal{S})$ such that $\mathcal{S}$ contains no stable set avoiding $Q^{*}$, then $G_{1}$ and $G_{2}$ are normal;

(2) If $G$ admits a valid pair $(\mathcal{Q}, \mathcal{S})$ such that $\mathcal{S}$ contains a stable set avoiding $Q^{*}$, then $G_{1}$ and $G_{2}$ are nearly normal with unnormal cliques $Q_{1}^{*}$ and $Q_{2}^{*}$ such that $Q_{i}^{*} \subseteq Q^{*}$ and $Q_{1}^{*} \cap Q_{2}^{*}=\emptyset$.

Note that in case (2) of the lemma, $Q_{1}^{*}$ and $Q_{2}^{*}$ may be empty (such that $G_{1}$ and $G_{2}$ become normal), but that the weaker condition that $G_{1}$ and $G_{2}$ are nearly normal already suffices, provided $Q_{1}^{*}$ and $Q_{2}^{*}$ are disjoint. However, we cannot obtain normal graphs by identifying two non-normal graphs in a node (as the unnormal cliques $Q_{1}^{*}, Q_{2}^{*} \subseteq Q^{*}$ cannot be disjoint if $\left|Q^{*}\right|=1$ ). We call a nearly normal graph almost normal if its unnormal clique consists in one node only. Except the $C_{5}$ (whose only possible unnormal clique has size two), all the graphs in Figure 4 are examples of almost normal graphs. We finally obtain the following result which is crucial for our purpose as an immediate consequence of Lemma 2:

Theorem 1 Construct a graph $G$ by identifying two graphs $G_{1}$ and $G_{2}$ in a node $q^{*}, G$ is normal if and only if one of the following conditions holds:

(1) $G_{1}$ and $G_{2}$ are normal;

(2) $G_{1}$ is normal and $G_{2}$ almost normal with unnormal node $q^{*}$, or vice versa.

Indeed, condition (1) of the theorem is satisfied if in Lemma 2 either case (1) occurs or case (2) with $Q_{1}^{*}$ and $Q_{2}^{*}$ empty; condition (2) of the theorem is satisfied if case (2) of Lemma 2 occurs where one of $G_{1}$ and $G_{2}$ is normal, the other is almost normal with unnormal clique $\left\{q^{*}\right\}=Q^{*}$.

\section{The Normal 1-Trees}

In this section we characterize the normal 1-trees, verify the Normal Graph Conjecture for such graphs, and provide a linear time recognition algorithm for normal 1-trees.

Recall that a 1-tree $G$ is a connected graph containing exactly one cycle $C$, i.e., $G$ can be obtained from the cycle $C$ and certain trees by a sequence of 
node-identifications. In order to characterize the normal 1-trees we use Theorem 1 and the result that a connected triangle-free graph is normal if and only if it has a so-called nice edge cover [9], as defined below.

Let $G=(V, E)$ be a graph and $\mathcal{F}$ be a minimal edge cover of $G$, i.e., an inclusion-wise minimal set $\mathcal{F} \subseteq E$ s.t. every node in $V$ is the endnode of some edge in $\mathcal{F}$ (note that a minimal edge cover is the union of node-disjoint stars). Consider a (not necessarily chordless) odd cycle $C$ in $G$ and the distribution of the edges of $\mathcal{F}$ alongside the cycle $C$. We say that a node $v$ of $C$ is even w.r.t. $\mathcal{F}$ if $v$ is the endnode of either none or two edges in $\mathcal{F} \cap E(C)$. Since $C$ is an odd cycle, $C$ has an odd number of even nodes. An edge cover of a graph $G$ is called nice if it is minimal and every odd cycle in $G$ has at least three even nodes.

For example, the bold edges of all three graphs in Figure 1 form nice edge covers, whereas the graphs in Figure 4 do not admit any nice edge cover.

Körner and de Simone showed in [9] that the occurrence of nice edge covers is sufficient for a graph to be normal. In particular, they characterized the triangle-free normal graphs as follows:

Theorem 2 [9] A connected triangle-free graph is normal if and only if it has a nice edge cover.

Let $G_{1}+{ }_{v} G_{2}$ denote the graph obtained from $G_{1}$ and $G_{2}$ by identification in the node $v$. For instance, the second graph in Figure 4 equals $C_{5}+{ }_{v} K_{2}$, obtained from a $C_{5}$ and a $K_{2}$ by node identification; the third graph in Figure 4 equals $\left(C_{5}+{ }_{v} K_{2}\right)+{ }_{v^{\prime}} K_{2}$, obtained from $C_{5}+{ }_{v} K_{2}$ and $K_{2}$ by identification in another node of the $C_{5}$.

We characterize the normal 1-trees with the help of Theorem 1 and Theorem 2 as follows.

Theorem 3 A 1-tree $G$ is not normal if and only if one of the following conditions holds.

(1) $G=C_{5}$.

(2) $G=C_{5}+{ }_{v} T$ where $T$ is a tree.

(3) $G=\left(C_{5}+{ }_{v} T\right)+{ }_{v^{\prime}} T^{\prime}$ where $T, T^{\prime}$ are trees and $v, v^{\prime}$ two non-consecutive nodes of the $C_{5}$.

(4) $G=C_{7}$.

Proof A 1-tree $G$ is obtained from a tree by adding one edge. Hence, $G$ contains exactly one chordless cycle $C$ and $G-C$ is a (possibly empty) forest. Thus, $G$ can be obtained from $C$ and certain trees by a sequence of node-identifications. We prove, dependent on the length of $C$, whether $G$ is normal or not.

Claim If $C \neq C_{5}, C_{7}$ then $G$ is normal.

Every hole $C \neq C_{5}, C_{7}$ is normal. Hence $G$ can be obtained from normal graphs by a sequence of node-identifications and is, thus, normal by Theorem 1(1). $\diamond$

It remains to consider the cases $C=C_{5}$ and $C=C_{7}$. Let $G_{5}$ denote the first graph depicted in Figure 1. 
Claim If $C=C_{5}$ then $G$ is normal if and only if $G$ contains the graph $G_{5}$ as subgraph.

$G$ is triangle-free in both cases, hence $G$ is normal if and only if it admits a nice edge cover by Theorem 2. Every minimal edge cover $\mathcal{F}$ of $G$ uses at most three edges of the $C_{5}$. All possible types of $\mathcal{F} \cap C_{5}$ are shown in Figure 5 (edges in $\mathcal{F} \cap C_{5}$ are drawn with bold lines, even nodes w.r.t. $\mathcal{F}$ are black-filled).

$\mathcal{F}$ is nice if and only if two consecutive nodes $v, v^{\prime}$ of the $C_{5}$ are not covered by the edges in $\mathcal{F} \cap C_{5}$ (the types (c), (d), and (e)). In order to cover the nodes $v, v^{\prime}$ by $\mathcal{F}$, there must exist non-empty trees $T, T^{\prime}$ identified with the $C_{5}$ in $v$ resp. $v^{\prime}$. Thus, $\mathcal{F}$ is nice if and only if $G$ contains the graph $G_{5}$ as subgraph. (Note that $G=C_{5}$ is not normal. If $G=C_{5}+{ }_{v} T$ or $G=\left(C_{5}+{ }_{v} T\right)+{ }_{v^{\prime}} T^{\prime}$ where $v, v^{\prime}$ are two non-consecutive nodes of the $C_{5}$, then $\mathcal{F}$ has to be of type (a) or (b) and is, therefore, not nice.) $\diamond$

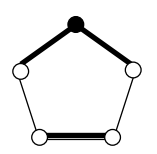

(a)

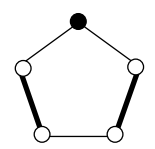

(b)

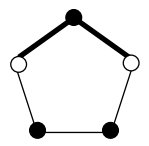

(c)

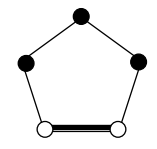

(d)

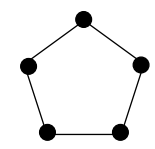

(e)

Fig. 5 Possible types of $\mathcal{F} \cap C_{5}$

Claim If $C=C_{7}$ then $G$ is normal if and only if $G \neq C_{7}$.

If $G=C_{7}$ then $G$ is clearly not normal. Otherwise, $G$ can be obtained from the $C_{7}$ and certain trees by a sequence of node-identifications. The $C_{7}$ is almost normal and all of its nodes are unnormal. Thus identifying the $C_{7}$ with a (nonempty) tree in a node yields a normal graph by Theorem 1(2), adding further trees maintains normality by Theorem $1(1)$. Hence $G$ is normal.

Thus, the non-normal 1-trees are all not $\left(C_{5}, C_{7}, \bar{C}_{7}\right)$-free by Theorem 3 which implies:

Corollary 2 The Normal Graph Conjecture is true for 1-trees.

As there are obviously normal 1-trees admitting a cycle of length 5 or 7 , we next address the question of recognizing normal 1-trees.

To find the cycle in a 1-tree $G=(V, E)$ as first step, we will use Algorithm LexBFS $[13]^{1}$. LexBFS is a specialized Breadth-First Search, which numbers

\footnotetext{
1 Although a simple DFS with a 'father' function could be used with the same time complexity, LexBFS provides us an elegant way to directly determine the studied cycle as well as its nodes.
} 
the nodes from $n=|V|$ to 1 ; each node $x$ obtains a label, denoted by lex $(x)$, which is the decreasing list of the neighbors of $x$ with a higher number than that of $x$. At each step, a node of lexicographically largest label is chosen to be numbered. A node will be referred to by its number, and when a node $x$ has a label of size 1, father $(x)$ will refer to the node in the label.

When the input graph has only one cycle, all the nodes obtain a label of size 1, except the node which 'closes' a cycle, whose label is of size 2. To find the corresponding cycle $C$, we can use the double label, which yields the next 2 nodes on $C$; we then go on to take the lowest numbered node between these two: its label yields the next node on $C$, and so on, until we find a node already in $C$, which will be the 'root' of $C$ in the tree induced by the LexBFS numbering. The algorithm can stop as soon as more than 7 nodes of the cycle have been found, as the graph is certain to be normal.

Theorem 4 Given a 1-tree $G=(V, E)$, Algorithm 1 decides in $O(|V|)$ whether or not $G$ is normal.

Proof Since $G$ is a 1-tree (i.e., $G$ is connected and has one cycle), Algorithm 1 finds at some point an already labeled neighbor $x$ of the current node $y$ and assigns a double label $l e x(x)=(y, z)$ (this can be done in $O(|V|)$ since at most $|V|=|E|$ edges have to be tested). The edge $x y$ closes a cycle $C$, and it remains to test whether $C$ has length 5 or 7 and the conditions of Theorem 3 are satisfied or not. For that, it suffices to go from $y$ and $z 5$ steps backwards along the unique paths towards the root node $r$ (always passing from a node to its father), looking for a common node of the paths: if $C$ has length 5 or 7 , it has been found that way, and the conditions of Theorem 3 decide about normality (which can be tested in constant time). In all other cases (i.e., if $C$ has length $3,4,6,8$ or no cycle has been found yet), $G$ is normal.

Remark 1 Algorithm 1 can be turned into a robust algorithm that in addition decides, still in linear time, whether the input graph $G=(V, E)$ is a 1-tree. For that, the graph has to satisfy $|V|=|E|$ and has to be connected. This can be tested by running BFS until no further nodes can be reached (then $G$ is a 1-tree if and only if $T$ contains all nodes from $V$ and exactly one edge $x y$ is not included).

\section{The Normal Cacti}

In this section we study cacti, i.e., graphs with edge-disjoint cycles. Since a cactus $G$ with $k$ cycles can be considered as a graph obtained from a tree by adding $k$ edges, we can alternatively obtain $G$ from $k 1$-trees by a sequence of node-identifications.

As we have characterizations of both normal graphs obtained by nodeidentification (Theorem 1) and normal 1-trees (Theorem 3) from the previous sections, it is natural to decompose a cactus $G$ accordingly: we have to choose 


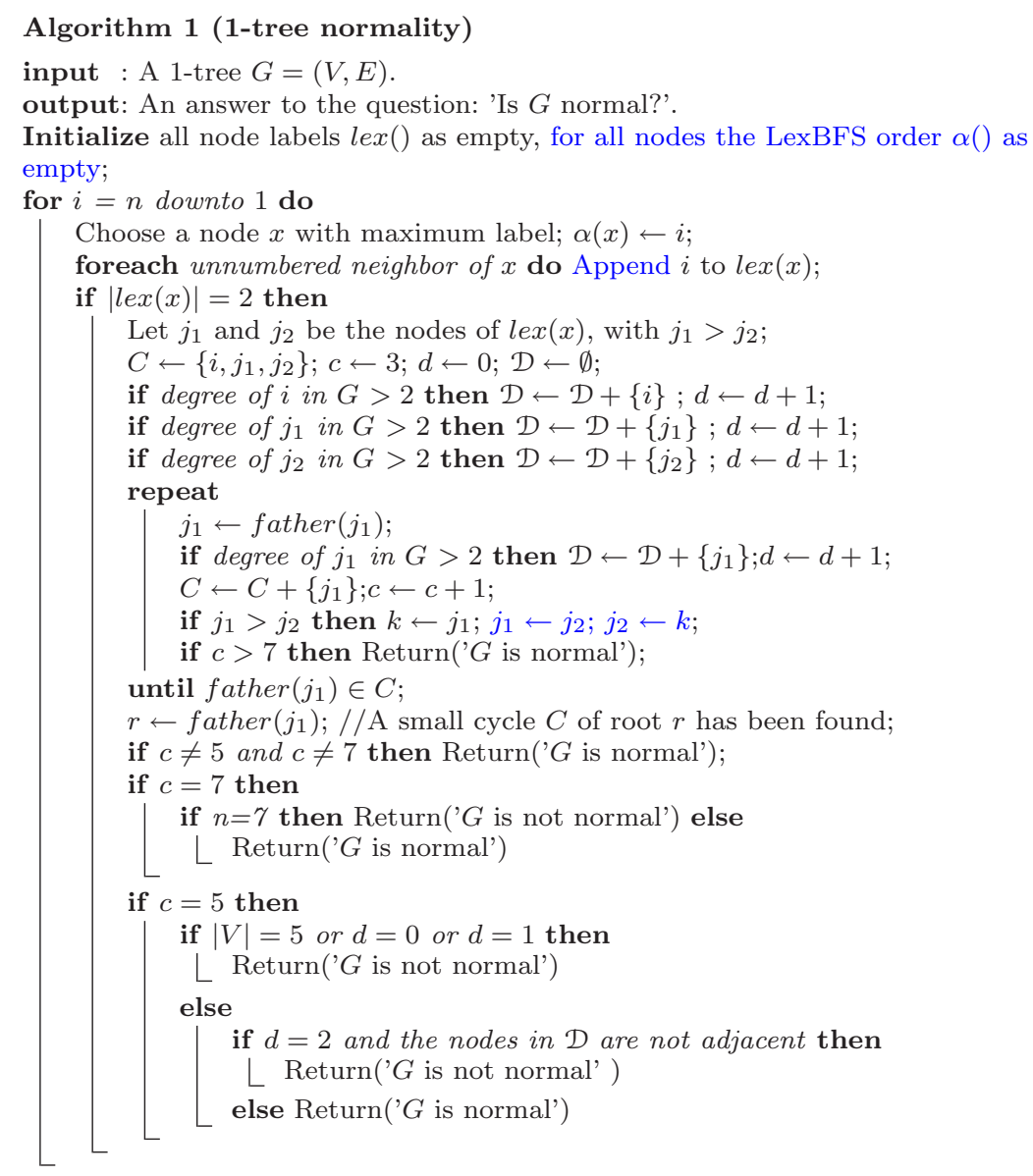

a set of articulation points in $G$ s.t. each building block contains exactly one cycle (and is, thus, a 1-tree). We denote the building block of cycle $C$ by $B(C)$.

If all these blocks $B(C)$ of $G$ are normal, then $G$ is clearly normal due to Theorem 1. This holds particularly if none of the cycles $C$ in $G$ has length 5 or 7 by Theorem 3; hence we have:

Corollary 3 The Normal Graph Conjecture is true for cacti.

As there are obviously normal cacti admitting a cycle of length 5 or 7 , our further goal is to find a way to decide whether or not a cactus is normal.

For that, we introduce the block-tree of a cactus as follows. For a cactus $G=(V, E)$ with $k$ cycles, we call a set $A \subseteq V$ of articulation points valid if $A$ can be used to decompose $G$ so that the resulting building blocks $B(C)$ contain exactly one cycle $C$ each.

Hence, using a valid set of articulation points decomposes a cactus in as many 1 -trees as it has cycles. We obtain the block-tree $T(G, A)=(A \cup \mathcal{B}, L)$ 
of $G$ by taking as nodes a valid set $A$ of articulation points and the set $\mathcal{B}$ of the resulting building blocks $B(C)$ of $G$ and joining two nodes of $T(G, A)$ if and only if one corresponds to an articulation point $q \in A$ and the other one to a block $B(C)$ with $q \in B(C)$.

By construction, $T(G, A)$ is a tree as it is bipartite and has no cycle. We call a leaf of $T(G, A)$ an endblock of $G$ and say that two blocks of $G$ are adjacent if they share an articulation point in $A$. Figure 6 shows a cactus $G$ and a block-tree $T(G, A)$ (the black nodes of $G$ are used as articulation points).
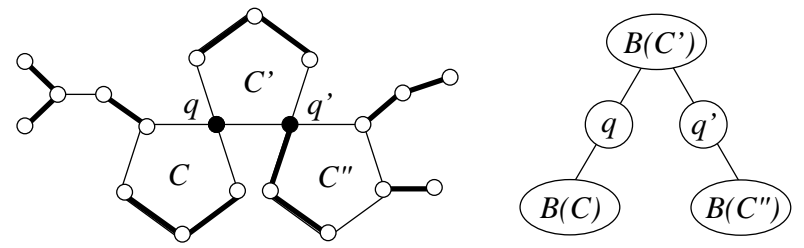

Fig. 6 A cactus and a block-tree

The main idea is to design an algorithm that, given a cactus $G$,

- finds a valid set $A \subseteq V$ and constructs the block-tree $T(G, A)$,

- decides the normality status of each block $B(C)$,

- shrinks $T(G, A)$ iteratively by deleting an endblock $B(C)$ and deciding how normal the remaining graph is.

We next explain in detail a procedure to construct $A$ and the block-tree $T(G, A)$, how to decide the normality status of each block $B(C)$, and of the remaining graph after deleting an endblock.

Constructing a block-tree To construct a block tree, we will again use algorithm LexBFS. A node will be referred to by its number. Each node will be assigned 2 labels: its LexBFS label lex $(x)$, and its block label block $(x)$ which is the list of block numbers which $x$ belongs to. At the end, block $(x)$ has size at least 2 if $x$ is in the set $A$ of valid articulation points. When a node $x$ has a label of size 1, father $(x)$ will refer to the node in the label.

In order to test for normality, we will also, while defining a cycle $C$, collect the set $\mathcal{C}$ of nodes of $C$ and the subset $\mathcal{D}$ of nodes of $C$ whose degree in $G$ is 3 or more. These information will be added to the block, as well as its status of 'normal' when it can be determined during the construction of the block tree. A Boolean firstcycle will avoid starting a new block when the very first cycle is encountered.

Theorem 5 Given a cactus $G=(V, E)$, Algorithm 2 constructs a block-tree of $G$ in $O(|V|)$.

Proof LexBFS, like any BFS, induces a spanning tree $T$ of the input graph $G$. When $G$ is a tree, $T$ is the same as $G$ and all the nodes have a label of size 0 or 


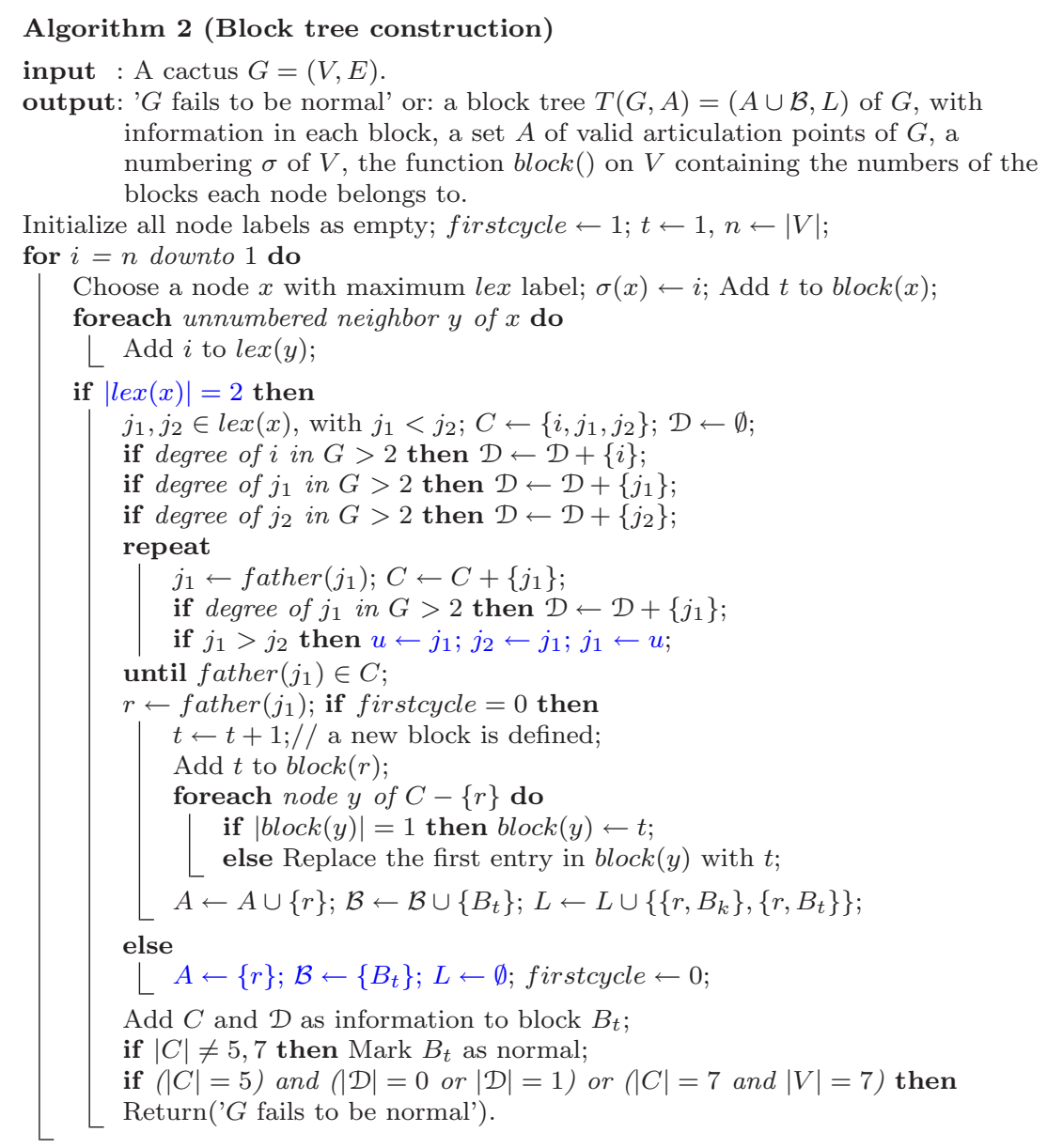

1. When $G$ is a cactus, the only edges of $G$ which are not in $T$ are those which close a cycle; clearly, these nodes will obtain a label of size 2 . Let us consider such a cycle $C$; the node $x$ with a double label is chosen to be numbered when all the nodes of $C$ have been numbered, thus $x$ is the lowest-numbered node of $C$; the highest-numbered node $y$ of $C$ is its root in $T$, and unless $y=n, y$ will be a valid articulation point, separating any neighbor $v$ of $y$ with $v>y$ from $C \backslash\{y\}$. (Note that $n$ may also be an articulation point, separating the nodes of two cycles).

When the label is not double, the newly numbered node is added to the highest-numbered block which its father belongs to. Algorithm 2 starts a new building block $B_{t}$ each time a cycle $C$ is closed and defined; the highestnumbered node is added to the block tree and to the set $A$ of valid articulation points, with the possible exception of node $n$; the block labels of the nodes of $C$ are updated: $t$ is added to the block label of the root of $C$; for the other 
nodes of $C$, if the node belongs to only 1 block, its block number is changed to the new block number $t$; if a node $v$ of $C$ belongs to more than 1 block, then its first block entry is changed to $t$ (by construction, the other block entries correspond to other blocks which $t$ is the root of).

The normality tests define as normal any block whose cycle is not of length 5 or 7 . The graph is found to be not normal when either the graph is a $C_{5}$ or a $C_{7}$, or when the current block contains a $C_{5}$ with only 1 neighbor.

LexBFS runs in linear time [13]; a cactus has $O(|V|)$ edges; defining the cycles costs at most $|V|$ as the cycle edges are traversed only once; thus Algorithm 2 runs in linear $O(|V|)$ time.

Deciding the normality status of blocks Since each block $B(C)$ is a 1-tree by construction, Theorem 3 characterizes whether it is normal or not. In view of Theorem 1, we have to distinguish the different not normal blocks: it is important to determine whether a not normal block $B(C)$ is almost normal and to specify its unnormal nodes.

For that, we denote the set of possible unnormal nodes of an almost normal block $B(C)$ by $U(C)$ and notice that all of them lie on $C$ : Any valid minimal clique cover of a cactus $G$ uses edges and triangles only, maintaining the notion of even nodes for odd cycles $C$ with length $\geq 5$. An unnormal node $q$ of $B(C)$ is not covered by the clique cover $\mathcal{Q}_{q}$ of a nearly valid pair $\left(\mathcal{Q}_{q}, \mathcal{S}\right)$ of $B(C)$, but it has to be covered by a clique outside $B(C)$ to become an even node of the cycle $C$.

As an immediate consequence of Theorem 3 and the possible nearly valid pairs of unnormal 1-trees in Figure 4, we obtain the following proposition.

Proposition 1 A block $B(C)$ with cycle $C$ is

(1) not (almost) normal if $B(C)=C_{5}$;

(2) almost normal with $U(C)=N(v) \cap C$ if $C=C_{5}$ and $B(C)=C_{5}+{ }_{v} T$ where $T$ is a tree;

(3) almost normal with $U(C)=C \backslash\left\{v, v^{\prime}\right\}$ if $C=C_{5}$ and $B(C)=\left(C_{5}+v\right.$ $T)+{ }_{v^{\prime}} T^{\prime}$ where $T, T^{\prime}$ are trees and $v, v^{\prime}$ are two non-consecutive nodes of the $C_{5}$;

(4) almost normal with $U(C)=C$ if $B(C)=C_{7}$;

(5) normal otherwise.

Proof As any block $B(C)$ is, by construction, a 1-tree, we distinguish according to Theorem 3 the following cases.

1. $C=C_{5}$ : Then $B(C)$ is normal if and only if $B(C)$ contains the graph $G_{5}$ as subgraph (due to the second claim in the proof of Theorem 3 ). We consider as subcases the three cases of Theorem 3 where $B(C)$ is not normal and check whether $B(C)$ is almost normal:

$1.1 B(C)=C_{5}$ : Then $B(C)$ is not almost normal (since the only possible unnormal clique of $C_{5}$ has size two, see Figure 4), which proves (1). 
$1.2 B(C)=C_{5}+{ }_{v} T$ where $T$ is a tree: Then $B(C)$ is almost normal and the two neighbors of $v$ are the possible unnormal nodes (see Figure 4), which proves $(2)$.

$1.3 B(C)=\left(C_{5}+{ }_{v} T\right)+{ }_{v^{\prime}} T^{\prime}$ where $T, T^{\prime}$ are trees and $v, v^{\prime}$ are two nonconsecutive nodes of the $C_{5}$ : Then $B(C)$ is almost normal and any neighbor of $v$ or $v^{\prime}$ is a possible unnormal node (see Figure 4), which proves (3).

2. $C=C_{7}$ : Then $B(C)$ is normal if and only if $B(C) \neq C_{7}$ by Theorem 3. If $B(C)=C_{7}$, then $B(C)$ is not normal, but almost normal and all its nodes are possible unnormal nodes (see again Figure 4), which proves (4).

3. $C \neq C_{5}, C_{7}$ : Then $B(C)$ is normal by Theorem 3 (as all not normal 1-trees contain $C_{5}$ or $\left.C_{7}\right)$.

Hence, (1)-(4) are exactly the cases when $B(C)$ is not normal (an if $B(C)$ is almost normal, the possible unnormal nodes are identified). In all other cases (i.e., if $C=C_{5}$ and $B(C)$ contains $G_{5}$, if $C=C_{7}$ but $B(C) \neq C_{7}$, or if $\left.C \neq C_{5}, C_{7}\right) B(C)$ is normal, which proves (5).

Shrinking a cactus by removing endblocks To iteratively shrink (the block-tree of) a cactus $G$ by removing an endblock $B(C)$, we use the following idea based on Theorem 1: If $B(C)$ does not satisfy any of the theorem's conditions, then $G$ is not normal. Otherwise, $G$ is normal if and only if the graph that results from $G$ by either removing $B(C)$ or collapsing a normal endblock $B(C)$ into a $K_{2}$ (i.e., into a smaller normal graph) is normal.

Consider a cactus $G$ and a block-tree $T(G, A)$. For an endblock $B(C)$ of $T(G, A)$ and (one of) its adjacent block(s) $B\left(C^{\prime}\right)$ with common node $q \in A$, we denote by $G-B(C)$ (resp. $G-B(C)+e$ ) the graph obtained by removing $B(C)$ maintaining $q$ (resp. by replacing $B(C)$ by an edge $e$ attached to $q$ ).

As an immediate consequence of Theorem 1 combined with Proposition 1 , we infer the normality of $G$ after removing $B(C)$ :

Corollary 4 Let $G$ be a cactus with block-tree $T(G, A), B(C)$ an endblock and $B\left(C^{\prime}\right)$ an adjacent block with common node $q \in A$.

(1) If $B(C)$ and $B\left(C^{\prime}\right)$ are normal, then $G$ is normal if and only if $G-B(C)$ is normal.

(2) If $B(C)$ is normal and $B\left(C^{\prime}\right)$ not, then $G$ is normal if and only if $G$ $B(C)+e$ is normal.

(3) If $B(C)$ is almost normal and $q \in U(C)$, then $G$ is normal if and only if $G-B(C)$ is normal.

(4) If $B(C)$ is almost normal and $q \notin U(C)$ or if $B(C)=C_{5}$, then $G$ is not normal.

While conditions (1), (3) and (4) are immediate consequences of Theorem 1 and Proposition 1, we note the following to justify condition (2): If $B(C)$ is normal but $B\left(C^{\prime}\right)$ not, then $G=B(C)+_{q}(G-B(C))$ is normal by Theorem 1 if and only if $G-B(C)$ is normal or almost normal with unnormal node $q$ 
(in $B\left(C^{\prime}\right)$ ). This is the case if and only if $K_{2}+_{q}(G-B(C))=G-B(C)+e$ is normal (which also follows from Theorem 1 since $K_{2}$ is normal so that the same conditions for $G-B(C)$ apply in both cases).

The above corollary enables us to shrink a cactus starting from endblocks, keeping normality of the input graph or deciding that $G$ is not normal. Thereby, the cases (1) and (3) allow us to simply remove $B(C)$, (4) provides sufficient conditions that $G$ is not normal, and (2) allows us to maintain or change the normality status of the remaining block $B\left(C^{\prime}\right)+e=K_{2}+{ }_{q} B\left(C^{\prime}\right)$ as follows:

Lemma 3 Let $G$ be a cactus with block-tree $T(G, A), B(C)$ a normal endblock and $B\left(C^{\prime}\right)$ an adjacent block with common node $q \in A$.

(1) If $B\left(C^{\prime}\right)$ is almost normal and $q \in U\left(C^{\prime}\right)$, then $B\left(C^{\prime}\right)+e$ is normal in $G-B(C)+e$.

(2) If $B\left(C^{\prime}\right)$ is almost normal and $q \notin U\left(C^{\prime}\right)$, then $B\left(C^{\prime}\right)+e$ remains almost normal with $U\left(C^{\prime}\right)$ in $G-B(C)+e$.

(3) If $B\left(C^{\prime}\right)=C_{5}$, then $B\left(C^{\prime}\right)+e$ is almost normal with $U\left(C^{\prime}\right)=N(q) \cap C^{\prime}$ in $G-B(C)+e$.

For the algorithmic process, however, it suffices to shrink the block-tree of a cactus by removing an endblock $B(C)$ and, if necessary, updating the normality status of $B\left(C^{\prime}\right)$ according to Lemma 3 .

Example 1 Reconsider the cactus $G$ and its block-tree $T(G, A)$ depicted in Figure 6 . Initially, we have the following normality status for its blocks:

- $B(C)$ is almost normal with $q \in U(C)$ (Proposition 1(2)),

- $B\left(C^{\prime}\right)=C_{5}$ is not normal (Proposition 1(1)),

- $B\left(C^{\prime \prime}\right)$ is normal (Proposition 1(5)).

We apply Algorithm 3 and notice that none of the sufficient conditions for $G$ being (not) normal is satisfied. Hence, we proceed with step "shrink" and select one of the two endblocks $B(C)$ and $B\left(C^{\prime \prime}\right)$ of $T(G, A)$.

If $B(C)$ is selected, then $B\left(C^{\prime}\right)$ is its only adjacent block with common node $q \in A$. None of the two conditions to update the normality status of $B\left(C^{\prime}\right)$ is satisfied, so we only remove $B(C)$ and $q$ from $T(G, A)$. The subsequent test for sufficient conditions reveals that $B\left(C^{\prime}\right)=C_{5}$ is now an endblock, hence the decision is "not normal" according to Corollary 4 (4).

On the other hand, if $B\left(C^{\prime \prime}\right)$ is selected, then $B\left(C^{\prime}\right)$ is its only adjacent block with common node $q^{\prime} \in A$. The second condition is satisfied, hence $B\left(C^{\prime}\right)$ is updated as almost normal with $U\left(C^{\prime}\right)=N\left(q^{\prime}\right) \cap C^{\prime} ; B\left(C^{\prime \prime}\right)$ and $q^{\prime}$ are removed from $T(G, A)$. The subsequent test for sufficient conditions reveals that now all blocks are not normal, hence the decision is "not normal" due to Theorem 1.

In both cases, the algorithm finds the correct answer "not normal".

Theorem 6 Given a cactus $G=(V, E)$, Algorithm 3 decides in $O(|V|)$ whether or not $G$ is normal. 


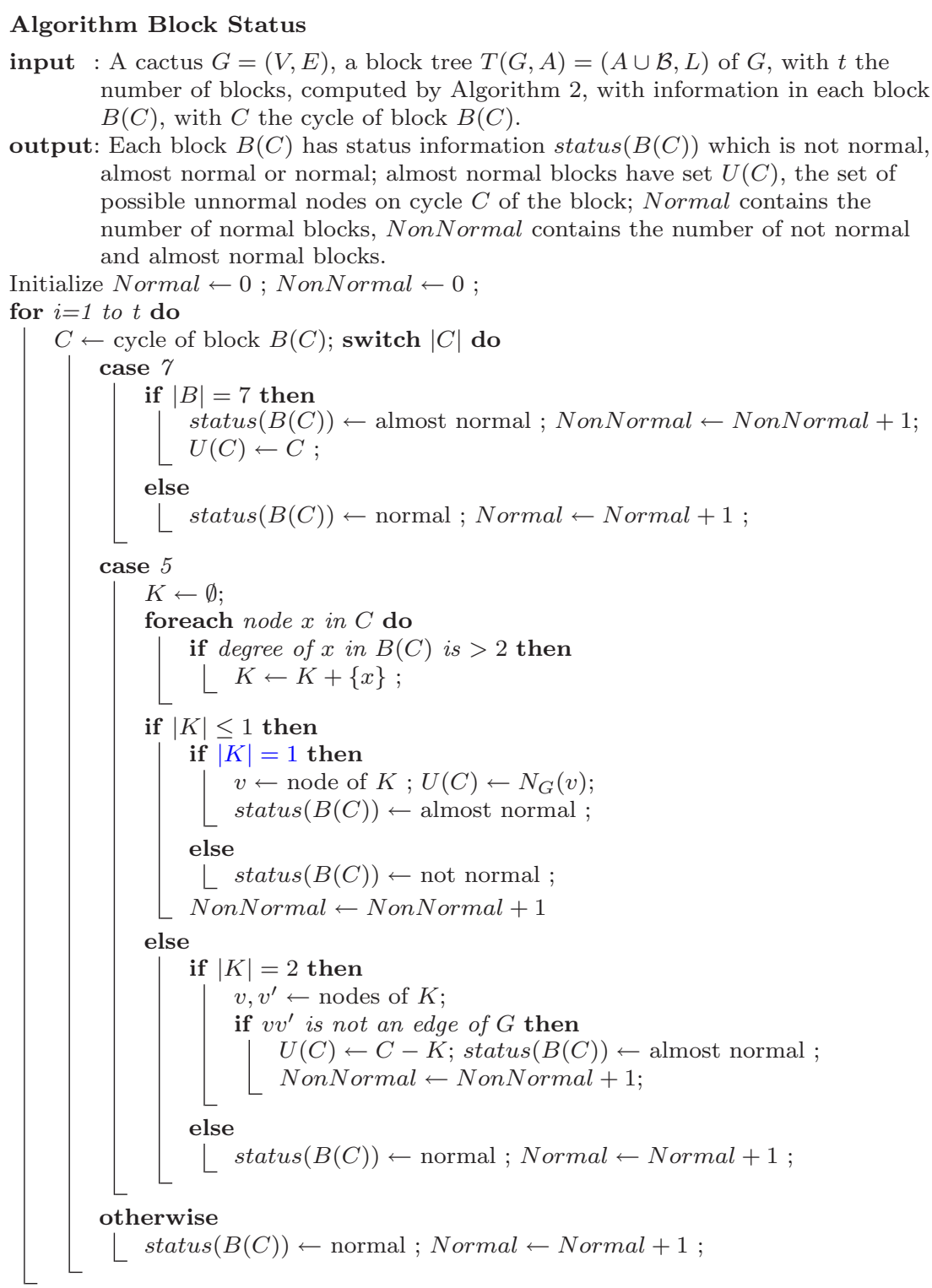

Proof For a given cactus $G$, Algorithm 2 constructs in linear time a valid set $A$ of articulation points and the corresponding block-tree $T(G, A)=(\mathcal{B} \cup$ $A, L)$. By construction, every block $B(C) \in \mathcal{B}$ is a 1 -tree, so the (easy to test) conditions of Proposition 1 determine its normality status (equals $C_{5}$ and is not normal, is almost normal with $U(C)$, is normal); Theorem 3 guarantees that all possible cases are considered. These tests can be performed at no extra cost while creating the blocks of $G$. 


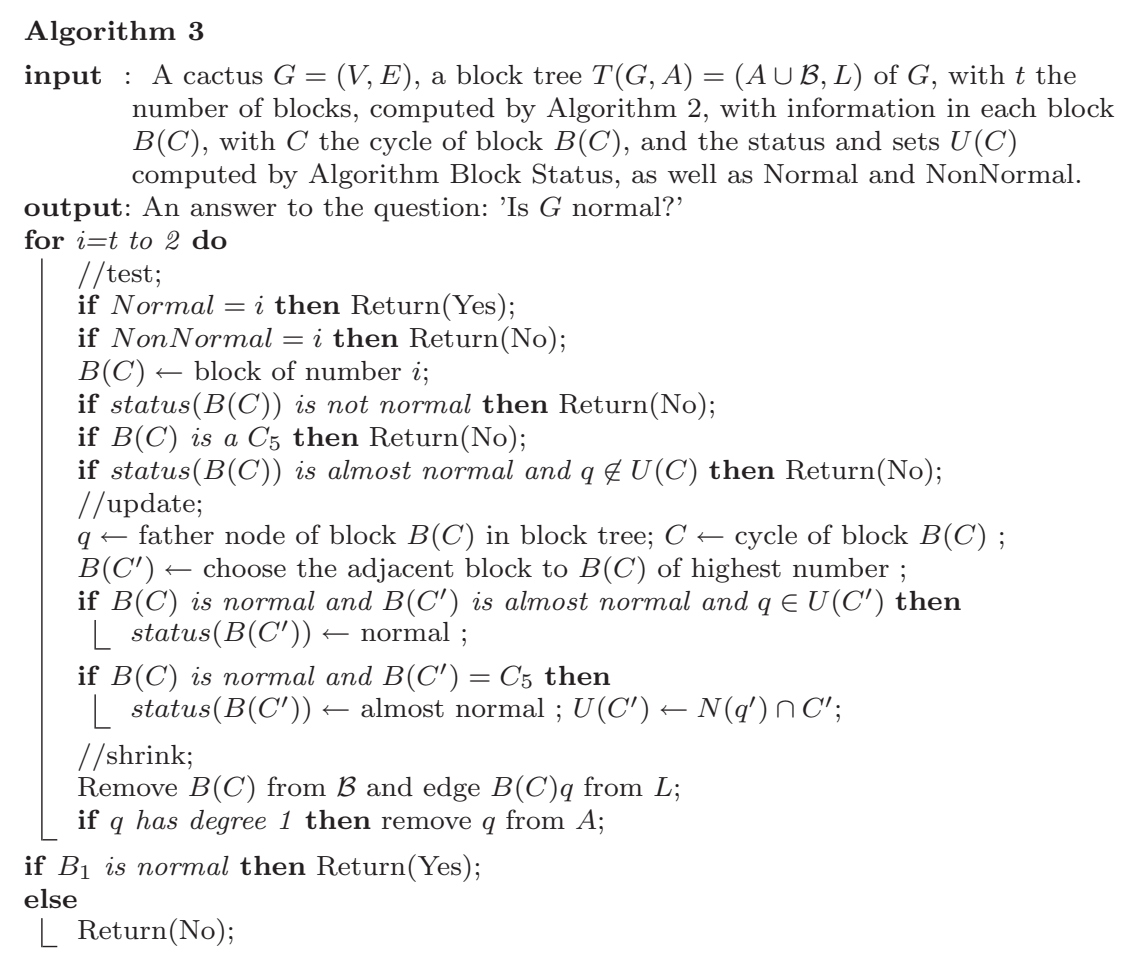

Next, if all blocks are normal (resp. not normal), then Theorem 1 guarantees that the whole graph is normal (resp. not normal). If one endblock $B(C)$ satisfies condition (4) of Corollary 4 (i.e., if $B(C)$ is almost normal and $q \notin U(C)$ or if $B(C)=C_{5}$ ), then $G$ is not normal (since both conditions of Theorem 1 fail).

If none of these sufficient conditions applies, it is necessary to choose an endblock $B(C)$; this is done by choosing the remaining block which was defined last by Algorithm 2, which is an endblock by construction as a depth-first search on a tree ends on a leaf; an adjacent block $B\left(C^{\prime}\right)$ is then chosen. Corollary 4 guarantees that the graph obtained by removing the endblock $B(C)$ is normal if and only if $G$ is normal. In the two tested cases, the normality status of the adjacent block $B\left(C^{\prime}\right)$ can be augmented according to Lemma 3 . Eventually, $B\left(C^{\prime}\right)$ becomes an endblock after removing $B(C)$.

At the end of step "shrink", we have the same situation as after the initial step and have to repeat both the tests for sufficient conditions and, if they fail, again step "shrink" for the next block.

The block-tree shrinks in each iteration, so that at latest after $|\mathcal{B}|-1$ iterations a decision can be made (as only one block is left). Corollary 4 and Lemma 3 guarantee that the current normality status of the remaining block corresponds to that of $G$. 
To efficiently perform the tests and the selection of an endblock, counters for the number of normal and not normal blocks are used. Then, step "shrink" defines an endblock and an adjacent block, performs two easy tests and updates the normality status of $B\left(C^{\prime}\right)$, if necessary. The tests for condition (4) of Corollary 4 has to be redone only for the new endblock. All this can be clearly done in linear time.

Finally, to obtain a characterization when a cactus $G$ is normal, recall that a triangle-free graph is normal if and only if it has a nice edge cover [9] and notice that triangles occur in a cactus only as or in normal blocks. This motivates the construction of a triangle-free graph $G_{\Delta}$ obtained from a cactus $G$ which is normal if and only if $G$ is:

Theorem 7 Let $G$ be a cactus and $G_{\Delta}$ be a graph obtained from $G$ by contracting exactly two nodes from each triangle of $G$. Then $G$ is normal if and only if $G_{\Delta}$ is normal if and only if $G_{\Delta}$ has a nice edge cover.

Proof Consider a cactus $G$ and a graph $G_{\Delta}$ constructed as above.

Claim 1. $G_{\Delta}$ is a triangle-free cactus.

In $G_{\Delta}$, none of the triangles of $G$ remains by construction. Since all cycles of $G$ are edge-disjoint, the construction does neither create new triangles, nor cycles sharing an edge. $\diamond$

By Claim 1, we can apply Algorithm 2 and 3 also to $G_{\Delta}$. In order to show that Algorithm 3 applied to $G$ and $G_{\Delta}$ returns the same output, we next consider the normality status of a block $B(C)$ with adjacent triangle $\Delta$ in $G$ and after the contruction in $G_{\Delta}$. We have:

Claim 2. Let $B(C)$ be a block and $\Delta$ be a triangle sharing a node $q$ with $B(C)$ in $G$ and $e$ be the edge in $G_{\Delta}$ obtained by contracting $\Delta$. Then $B(C)+{ }_{q} \Delta$ is as normal as $B(C)+e$ and the sets of unnormal nodes are equal.

This can be easily seen by simple case analysis for all possible normality status of $B(C)$ according to Proposition 1 and observations as in Corollary 4 and Lemma 3. $\diamond$

Now, apply Algorithm 3 to $G$ and $G_{\Delta}$. Notice that the obtained blocktrees can be equal only if $G$ is triangle-free; otherwise $T\left(G_{\Delta}, A_{\Delta}\right)$ contains less blocks than $T(G, A)$.

For the test of sufficient conditions, we have: On the one hand,

- if all blocks of $G$ are normal, then all blocks of $G_{\Delta}$ are normal;

- if all blocks of $G_{\Delta}$ are normal, but not all of $G$, then for each not normal block $B(C)$ of $G$, there is an attached triangle $\Delta$ such that combining $B(C)$ and $\Delta$ yields a normal block. 
In both cases, the output is "normal" for $G$ and $G_{\Delta}$. On the other hand,

- if all blocks of $G$ are not normal, then $G$ is triangle-free and $G=G_{\Delta}$;

- if all blocks of $G_{\Delta}$ are not normal, but not all of $G$, then each triangle $\Delta$ of $G$ is attached to a not normal block $B(C)$ such that combining $B(C)$ and $\Delta$ in yields a not normal block;

- if an endblock of $T(G, A)$ satisfies Corollary 4.(4), so does a corresponding endblock of $T\left(G_{\Delta}, A_{\Delta}\right)$.

In all cases, the output is "not normal" for $G$ and $G_{\Delta}$.

Finally, contracting two nodes of a triangle can be seen as shrinking $T(G, A)$ and Claim 2 guarantees that the outcome is the same. This proves that $G$ is normal if and only if $G_{\Delta}$ is, and the remaining assertion follows from [9] since $G_{\Delta}$ is triangle-free.

Remark 2 For a given cactus $G$, the triangle-free cactus $G_{\Delta}$ is not unique as there are three choices for the contruction of two nodes per triangle $\Delta$ in $G$. Similarly, during the process of Algorithm 3, neither the construction of the block-tree $T(G, A)$ of $G$ is unique (due to multiple choices for $A$ ), nor the shrinking order (due to multiple choices for end-blocks and their adjacent blocks).

However, for each $T(G, A)$ and shrinking order of $G$, there is a corresponding graph $G_{\Delta}$, obtained by contracting the two nodes in the selected triangle $\Delta$ at hand which are not in common with the other selected block. Then, contracting these two nodes of $\Delta$ to obtain $G_{\Delta}$ clearly equals a shrinking step for $T(G, A)$, and the outcome of both proceedures is clearly the same.

\section{Concluding Remarks}

In this work, we verify the Normal Graph Conjecture for two classes of sparse graphs, 1-trees and cacti. This shows that no counterexample to the Normal Graph Conjecture can be found in any of these classes (i.e. no cactus with girth at least 8 can be such a counterexample). Moreover, we solve the problem of deciding whether a 1-tree or a cactus is normal even when it does contain a $C_{5}$ or a $C_{7}$.

Finally, it would be interesting to generalize our techniques and results to larger graph classes. Canonical candidates are superclasses of cacti, e.g., chordless graphs (whose cycles are all chordless) or outerplanar graphs (who admit an embedding into the plane such that no edges cross and all nodes lie on the outer face). The question is to find suitable decompositions for such graphs into blocks such that the normality status of both the blocks and the recomposed graphs can be determined.

In this context, our future line of research will be to decompose an outerplanar graph into 1-trees with the help of clique separators of size 1 and 2, to characterize normal graphs obtained by identifying two graphs in a clique of size 2 (in analogy to Theorem 1), and to deduce results similar to Corollary 4 and Lemma 3 for deciding the normality status of the graph obtained from 
removing endblocks of such a decomposition. This would enable us to verify or falsify the Normal Graph Conjecture for outerplanar graphs and to recognize normal outerplanar graphs in a similar way as normal cacti.

\section{References}

1. C. Berge, Färbungen von Graphen, deren sämtliche bzw. deren ungerade Kreise starr sind, Wiss. Zeitschrift der Martin-Luther-Universität Halle-Wittenberg 10 (1961) 114115 .

2. A. Berry, A.K. Wagler, The Normal Graph Conjecture for classes of sparse graphs, Lecture Nodes in Computer Science 8165 (2013) 64-75 (Special Issue WG 2013)

3. M. Chudnovsky, N. Robertson, P. Seymour, and R. Thomas, The Strong Perfect Graph Theorem, Annals of Mathematics 164 (2006) 51-229.

4. I. Cziszár, J. Körner, L. Lovász, K. Marton, and G. Simonyi. Entropy splitting for antiblocking corners and perfect graphs, Combinatorica 10 (1990) 27-40.

5. A. Harutyunyan, L. Pastor, and S. Thomassé. Disproving the Normal Graph Conjecture, arXiv:1508.05487v5 (math.CO)

6. J. Körner, An Extension of the Class of Perfect Graphs, Studia Math. Hung. 8 (1973) 405-409.

7. J. Körner and G. Longo, Two-step encoding of finite memoryless sources, IEEE Trans. Inform. Theory 19 (1973) 778-782.

8. J. Körner and K. Marton, Graphs that split entropies, SIAM J. Discrete Math. 1 (1988) $71-79$.

9. J. Körner and C. de Simone, On the Odd Cycles of Normal Graphs, Discrete Appl. Math. 94 (1999) 161-169.

10. L. Lovász, Normal Hypergraphs and the Weak Perfect Graph Conjecture, Discrete Math. 2 (1972) 253-267.

11. H.J. Prömel and A. Steger, Almost all Berge graphs are perfect, Combinatorics, Probability, and Computing 1 (1992) 53-79.

12. H.O. Schülzke, The Normal Graph Conjecture for line graphs. Diplomarbeit, TU Berlin, 2006.

13. D.J. Rose, R.E. Tarjan, and G.S. Lueker. Algorithmic aspects of vertex elimination on graphs. SIAM J. Comput., 5:266-283, 1976.

14. C.E. Shannon. The zero-error capacity of a noisy channel, IRE Trans. Inform. Theory 2 (1956) 8-19.

15. G. Simonyi. Perfect Graphs and Graph Entropy: An Updated Survey, In: Perfect Graphs, J.L. Ramirez-Alfonsin und B.A. Reed (eds.), Wiley, pages 293-328, 2001.

16. A.K. Wagler, The Normal Graph Conjecture is true for circulants, In: Graph Theory in Paris, A. Bondy et al. (eds.) Trends in Mathematics, Birkhäuser, Basel (2007) 365-374.

17. A.K. Wagler, Constructions for Normal Graphs and Some Consequences, Discrete Applied Mathematics 156 (2008) 3329-3338. 


\section{Reply to the referee}

We would like to thank the referee for the careful reading of the paper and the suggestions made concerning corrections of the algorithms and the clarity of the presentation.

We have handled the comments as follows (all changes made are highlighted in blue within the file "BW_rev-highlighted.pdf").

1. we removed the case of tringle-free graphs here

2. this result was obtained in a master thesis - we don't know what could help to reference or access it

3. we added " $C_{3}$ and" (note that $C_{4}$ is indeed a subgraph of $\bar{C}_{7}$ )

4. done

5. indeed, $Q_{1}^{*}$ or $Q_{2}^{*}$ can be empty (s.t. $G_{1}$ or $G_{2}$ becomes normal); we added an explanation after Lemma 2

6. the theorem is indeed an immediate consequence of Lemma 2 (in the special case when $Q^{*}$ is a single node $q^{*}$ ); we did not add a formal proof, but an explanation how Lemma 2 is applied in this special case

7. done

8. done

9. done (the first if/else block is replaced by a single if condition)

10. done

11. done

12. this fact directly follows from Theorem 1 (an explanation is added how)

13. done

14. the meaning of the sentence is: the (easy to test) conditions of Proposition 1 determine its normality status

15. done

16. done

17. done 